Y.-H. Chu, N.B. Suntzeff, J.E. Hesser, and D.A. Bohlender, eds.

\title{
On the Period-Luminosity-Color Relation of Classical Cepheids
}

\author{
G. Bono ${ }^{1}$ \\ Osservatorio Astronomico di Roma, Via Frascati 33, 00040 \\ Monteporzio, Italy \\ M. Marconi ${ }^{2}$ \\ Osservatorio Astronomico di Napoli, Via Moiariello 16, 80100 Napoli, \\ Italy
}

\begin{abstract}
.
We compare the theoretical and empirical $\mathrm{PL}$ relations in $\mathrm{V}$ and $\mathrm{I}$ bands for Cepheids in the LMC. We found that, within the current intrinsic dispersions, the theoretical predictions are in remarkable agreement with observational data. We also discuss the PLC relations in (V,B-V) and $(\mathrm{V}, \mathrm{V}-\mathrm{I})$ as well as the dependence of distance determinations on uncertainties in colors, in reddening corrections and in metal content.
\end{abstract}

\section{Introduction}

Classical Cepheids are the most popular primary distance indicators. The key role played by this group of radial variables in estimating the cosmic distance scale is soundly confirmed by the large number of both observational and theoretical investigations aimed at improving both accuracy and reliability of distance measurements. At least three are the main reasons for the widespread use of the Cepheid distance scale:

- Cepheids are bright intermediate-mass objects with visual magnitude range from $M_{V}=-3$ at short-periods $(\log P \approx 0.6)$ to $M_{V}=-6$ at long-periods $(\log P \approx 2.0)$. This feature and the luminosity variations over the pulsation cycle make Cepheids an appealing observational target, since they can be detected and measured in a large number of Local Group galaxies. With the HST, the distance scale key project has identified a good number of Cepheirds in two dozens galaxies out to the Virgo cluster.

- The physical mechanisms which govern the pulsational instability of these objects have been firmly established. This notwithstanding, the pulsation behavior and the modal stability of Cepheids are often outlined by adopting näive arguments. Moreover, up to now no general theoretical consensus on the dependence of the Cepheid luminosity on the metal abundance has been reached.

\footnotetext{
${ }^{1}$ On leave from Osservatorio Astronomico di Trieste, Via G.B. Tiepolo 11, 34131 Trieste, Italy

${ }^{2}$ Dipartimento di Fisica Università di Pisa, Piazza Torricelli 2, 56126 Pisa, Italy
} 
In fact, theoretical predictions based on linear, nonadiabatic, radiative models suggest that the blue edge of the instability strip presents a negligible dependence on metal content (Saio \& Gautschy 1998). On the other hand, recent theoretical predictions which account for both blue and red edges of the instability strip support the evidence that Cepheid luminosity depends on chemical composition. In particular, it turns out that metal-poor Cepheids are, at fixed period, brighter than metal-rich ones. This prediction is at odds with their empirical behavior, and indeed a trend opposite to this has recently appeared in the literature (Sasselov et al. 1997; Kennicutt et al. 1998 and references therein).

- Some of the long-standing questions on distance determinations can be properly addressed within the Cepheid scenario. The intrinsic width of the instability strip is substantially narrower in near infrared (NIR) bands than in optical. Metallicity dependence and reddening corrections give the same outcome, and indeed NIR PL relations are less affected by these uncertainties than optical PL relations. The calibration of the PL zero-points can be accomplished by adopting several independent methods such as the Baade-Wesselink method and its progeny (Krockenberger et al. 1997; Di Benedetto 1997), the trigonometric parallaxes (Feast \& Catchpole 1997), and the main sequence fitting (Gieren \& Fouquè 1993). Therefore a straightforward analysis of both systematic and intrinsic errors affecting distance determinations may be undertaken.

Cepheids are the primary extragalactic distance indicators, and affect directly the evaluation of the Hubble constant $-H_{0}$. As noted by Trimble (1997), over the years $H_{0}$ estimates decreased by one order of magnitude, but the error bars remained almost constant! Clearly, we need a comprehensive theoretical investigation of the deceptive errors which could affect distance measurements.

In $\S 2$ we discuss the comparison between theoretical and empirical PL relations in $\mathrm{V}$ and $\mathrm{I}$ bands for Cepheids in the Large Magellanic Cloud (LMC), as well as the so called Wesenheit function. Theoretical prediction concerning the Period-Luminosity-Color (PLC) relations are presented in $\S 3$ together with a brief analysis of the uncertainties due to both metallicity and reddening.

\section{PL Relations for LMC Cepheids}

LMC Cepheids are the backbone of cosmic distance derivation since the PL relations of the target galaxy are compared with the LMC PL relations when placing extragalactic distances on an absolute scale. Even though the Cepheid metallicity is not firmly constrained (see Luck et al. 1998) and the reddening estimates still present some uncertainties, LMC Cepheids cover a wide period range and are relatively close objects, thus enabling a proper sampling of the instability strip.

In a recent paper Tanvir (1997) performed a thorough analysis of the intrinsic and systematic uncertainties which affect Cepheid distance scale. In particular, the author derived new LMC PL relations based on current available data in $\mathrm{V}$ and $\mathrm{I}$ bands, as well as a $\mathrm{PL}$ relation for the reddening free Wesenheit function, i.e. $W_{V I}=V-R[V-I]$ where $R \approx 2.45$ is the adopted extinction parameter (Cardelli et al. 1989). These empirical relations were calibrated by adopting an LMC distance modulus of 18.5 and a reddening of $E_{B-V}=0.1 \mathrm{mag}$, respectively. In order to supply a theoretical framework for a 
proper comparison with observational data we derived analytical PL relations by adopting several sequences of Cepheid models constructed with fixed chemical composition ( $\mathrm{Y}=0.25 \mathrm{Z}=0.008$ ) and a wide range of stellar masses and effective temperatures (see Bono et al. 1999a,b for further details). The mass-luminosity relation adopted for fixing the luminosity of these models is based on evolutionary tracks which neglect the convective core overshooting during the hydrogen burning phase.

In deriving these relations we anchored the period at $\log P=1.4$. We did not restrict the period range to $\log P<1.8$ (Tanvir 1997), since we are interested in testing theoretical predictions for long-period Cepheids. Due to the well known bending of the PL relation in the long-period range we performed a quadratic fit and the results we obtained are the following:

$$
\begin{array}{lllll}
\left\langle M_{V}\right\rangle= & -5.18 & -2.01[\log P-1.4] & +0.88[\log P-1.4]^{2} & \left(\sigma_{r m s}=0.25\right) \\
\left\langle M_{I}\right\rangle= & -6.09 & -2.43[\log P-1.4] & +0.67[\log P-1.4]^{2} & \left(\sigma_{r m s}=0.18\right) \\
\left.<W_{V I}\right\rangle= & -7.41 & -3.02[\log P-1.4] & +0.38[\log P-1.4]^{2} & \left(\sigma_{r m s}=0.08\right)
\end{array}
$$

Figure 1 shows the comparison between theoretical and empirical PL relations. From this comparison three interesting results emerge: 1) In the period range covered by empirical relations $(0.4<\log P<1.7)$ the mean $P L_{V}$ and $P L_{I}$ relations are, within the intrinsic dispersions, in remarkable agreement with theoretical predictions. 2) The theoretical PL relations show a linear behavior up to $\log P \approx 1.6$ but toward longer periods they start to bend due to the shift of the instability strip toward redder colors. 3) Theoretical and empirical dispersions in the I band are, as expected, smaller than those of the $\mathrm{V}$ band, but they are still too large for constraining the distance modulus and the reddening values adopted for calibrating this sample. This notwithstanding, there is no plausible reason for assuming that the pulsation characteristics of LMC Cepheids are peculiar (Simon \& Young 1997), thus supporting the use of these templates for estimating the absolute distance of target galaxies with similar metal contents.

The bottom panel of Figure 1 shows the comparison between theoretical and empirical $P L_{W}$ relations based on Wesenheit magnitudes. The two relations are in agreement only marginally, and when moving from long to short-periods the discrepancy increases. Due to the agreement between theory and observations in the $\log P-M_{V}$ and in the $\log P-M_{I}$ plane, it is not clear whether this drift is caused by a systematic shift in the color-temperature relations we adopted or more likely is caused by a poor accuracy of (V-I) mean colors. In fact, in order to reduce the sampling of I light curves the $\mathrm{V}$ band light curves are transformed into I band light curves by adopting an empirical method (see Appendix $A$ in Tanvir 1997). We note that the light curves are not characterized by a constant shape when moving from short to long-period Cepheids. In the short-period range they present a sawtoothed shape, then around $\log P \approx 1$ they show a bump along either the rising or the decreasing branch while in the long-period range the shape becomes more sinusoidal.

It has been often pointed out that the use of these magnitudes causes a substantial decrease in the dispersion of the PL relation. This is mainly due to the fact that the Wesenheit function in two arbitrary bands $(\beta, \xi)$ is the projection of the $\operatorname{PLC}(\beta, \beta-\xi)$ relation onto the $\log P-M_{\beta}$ plane. As a consequence, in this plane the dispersion is much smaller since the location of 


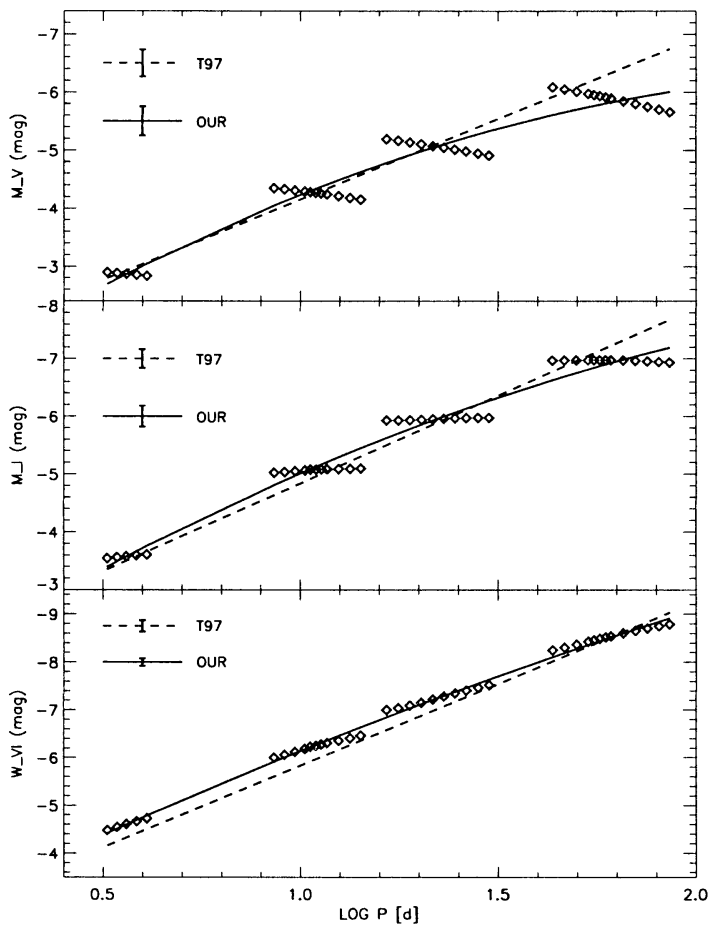

Figure 1. Comparison between our theoretical PL relations (solid lines) and the empirical relations (dashed lines) provided by Tanvir (1997). The error bars are referred to the intrinsic dispersions of analytical relations. From top to bottom the relation plotted in this figure are the $P L_{V}$, the $P L_{I}$ and the Wesenheit function.

each individual Cepheid inside the instability strip is being fixed according to both period and color.

Is the Wesenheit Function Equivalent to Using a PLC Relation for Estimating Distances?

On general grounds the answer is No, because the Wesenheit function is only mimicking the behavior of the equivalent PLC relation. Due to coincidence, the value of the color coefficient in the optical PLC (V, B-V) relation is quite similar (see Bono et al. 1999a) to the extinction parameter, and thus in these bands the Wesenheit function and the PLC relation present the same behavior. However, the Wesenheit function $(\mathrm{V}, \mathrm{I})$ is derived by adopting $R \approx 2.45$ (Cardelli et al. $1989)$ and therefore it is quite different from the color coefficient of the PLC (V, $\mathrm{V}-\mathrm{I}$ ) relation (see below).

On the basis of the above results three main conclusions concerning the use of the $\log P-W_{V I}$ relation can be drawn: 1) in these bands the Wesenheit function does not mimic the behavior of the PLC (V, V-I) relation and therefore it does not properly account for the intrinsic width of the instability strip. 2) This relation is sensitive to the photometric accuracy of mean colors and therefore for a sound empirical evaluation a good sampling of both $\mathrm{V}$ and I band light 
curves is needed. This means that if accurate mean colors are available the use of the PLC (V, V-I) relation for estimating distances is more physically plausible than the Wesenheit function. The interesting feature that the Wesenheit function supplies reddening free magnitudes will be discussed in a forthcoming paper (Caputo et al. 1999).

\section{Discussion and Conclusions}

The above discussion has been focused on the PL relations at fixed chemical composition. In order to account for the dependence of distance determinations on metallicity we adopt the PLC relations, since in this parameter space the Cepheid location is not ambiguous. We derived two analytical relations by taking into account sequences of models constructed by adopting three different chemical compositions, namely $\mathrm{Y}=0.25, \mathrm{Z}=0.004 /[\mathrm{Fe} / \mathrm{H}]=-0.7 ; \mathrm{Y}=0.25$, $\mathrm{Z}=0.008 /[\mathrm{Fe} / \mathrm{H}]=-0.4 \mathrm{Y}=0.25, \mathrm{Z}=0.02 /[\mathrm{Fe} / \mathrm{H}]=0.0$. The results of this fit for $(\mathrm{V}, \mathrm{B}-\mathrm{V})$ and $(\mathrm{V}, \mathrm{V}-\mathrm{I})$ PLC relations are the following:

$$
\begin{array}{lllll}
\left\langle M_{V}\right\rangle= & -2.83 & -3.57 \log P & +2.92[\langle B\rangle-\langle V\rangle] & -0.35[\mathrm{Fe} / \mathrm{H}] \\
& \pm 0.05 & \pm 0.03 & \pm 0.05 & \pm 0.03 \\
& & & \\
<M_{V}>= & -3.57 & -3.59 \log P & +3.80[\langle V\rangle-<I\rangle] & +0.03[\mathrm{Fe} / \mathrm{H}] \\
\pm 0.03 & \pm 0.02 & \pm 0.04 & \pm 0.02
\end{array}
$$

where the symbols have their usual meaning. The standard deviations of these two relations are $\sigma_{r m s}=0.05$ and 0.03 respectively. As already pointed out by Bono et al. (1999a) the PLC (V, B-V) relation suggests that metal-rich Cepheids are, at fixed period and color, brighter than metal-poor ones. On the other hand, the PLC (V, V-I) relation discloses a marginal dependence on metallicity, and thus leads strong support to the use of this relation for estimating distances of target galaxies for which the metal content is poorly known.

Since these relations are not affected by systematic errors such as reddening corrections, photometric accuracy of both magnitude and colors, and the metal content, we can estimate how plausible errors within these parameters may affect distance determinations. At first, to account for photometric and zero-point calibration uncertainties we assumed, by following Tanvir (1997), that $\sigma_{B} \approx$ $\sigma_{V} \approx \sigma_{I} \approx 0.04 \mathrm{mag}$. These uncertainties imply errors on (B-V) and (V-I) colors of the order of $0.06 \mathrm{mag}$, and in turn an uncertainty in distance of $8 \%$ and $10 \%$. At the same time, a systematic error of the order of $0.03 \mathrm{mag}$ in the reddening correction $-E_{B-V^{-}}$implies errors on (B-V) and (V-I) colors of 0.03 and $0.04 \mathrm{mag}$ respectively. These uncertainties imply errors in distance equal to $4 \%$ and $7 \%$. However, if we simultaneously account for the two quoted uncertainties, the errors on the colors are equal to 0.19 and $0.26 \mathrm{mag}$, while in distances the errors are $9 \%$ and $12 \%$ respectively.

In order to account for the dependence of distance determinations on chemical composition we assumed an uncertainty on metal abundance of the order of 0.4 dex. This error takes into account not only the intrinsic errors in metallicity measurements but also the spread in metallicity of Cepheids in external galaxies. In fact, Monteverde et al. (1997) by adopting spectroscopic data of B-type supergiants in M33 estimated that the iron abundance gradient in this spiral 
galaxy is of the order $-0.20( \pm 0.05)$ dex $\mathrm{kpc}^{-1}$ The quoted uncertainty implies errors in distance modulus of $0.14(\mathrm{~V}, \mathrm{~B}-\mathrm{V})$ and of $0.012(\mathrm{~V}, \mathrm{~V}-\mathrm{I}) \mathrm{mag}$ and thus errors of $7 \%$ and $1 \%$ respectively.

The main outcome of this leading term error analysis are the following: 1) PLC relations based on different photometric bands present pros and cons, and indeed the PLC $(\mathrm{V}, \mathrm{B}-\mathrm{V})$ relation, in comparison with the PLC $(\mathrm{V}, \mathrm{V}-\mathrm{I})$ relation, is less affected by errors on both reddening corrections and colors but is more affected by a spread in metallicity. 2) The PLC (V,V-I) relation seems a promising distance indicator for target galaxies with accurate reddening and color measurements since it is marginally affected by metallicity.

Obviously a comprehensive analysis of the error budget of Cepheid distance scale based on PLC relations is not a trivial effort, since in reality it is a mixture of all previous uncertainties. A thorough analysis can be undertaken only by means of Monte Carlo simulations of Cepheids inside the instability strip, which can simultaneously account for all plausible errors on observables.

Acknowledgments. This work was partially supported by CNA through a postdoc research grant to $\mathrm{M}$. Marconi, by ASI and CRA.

\section{References}

Bono, G., Caputo, F., Castellani, V., \& Marconi, M. 1999a, ApJ, 512, 711

Bono, G., Marconi, M., \& Stellingwerf, R. F. 1999b, ApJS, 122, 167

Caputo, F., et al. 1999, in preparation

Cardelli, J. A., Clayton, G. C., \& Mathis, J. S. 1989, ApJ, 345, 245

Di Benedetto, G. P. 1997, ApJ, 486, 60

Feast, M. W. 1984, in IAU Symp. 108, Structure and Evolution of the Magellanic Clouds, eds. S. van der Bergh \& K.S. de Boer, Dordrecht: Kluwer, 157

Feast, M. W. 1991, in Observational Tests of Cosmological Inflation, eds. T. Shanks et al., Dordrecht: Kluwer, C348, 147

Feast, M. W. 1995, in IAU Colloq. 155, Astrophysical Applications of Stellar Pulsation, eds. R.S. Stobie \& P.A. Whitelock, San Francisco: ASP, 209

Feast, M. W., \& Catchpole, R. M. 1997, MNRAS, 286, L1

Gieren, W. P., \& Fouquè, P. 1993, AJ, 106, 734

Kennicutt, R. C., et al. 1998, ApJ, 498, 181

Krockenberger, M., Sasselov, D. D., \& Noyes, R. W. 1997, ApJ, 479, 875

Luck, R. E., Moffett, T. J., Barnes, T. G., \& Gieren, W. P. 1998, AJ, 115, 605

Monteverde, M. I., et al. 1997, ApJ, 474, L107

Saio, H., \& Gautschy, A. 1998, ApJ, 1998, 498, 360

Sasselov, et al. 1997, A\&A, 324, 471

Simon, N. R., \& Young, T.S. 1997, MNRAS, 288, 267

Tanvir, N. R. 1997, in The Extragalactic Distance Scale, eds. M. Livio, M. Donahue \& N. Panagia, Cambridge: Cambridge Univ. Press, 91

Trimble, V. 1997, in The Extragalactic Distance Scale, eds. M. Livio, M. Donahue \& N. Panagia, Cambridge: Cambridge Univ. Press, 313 


\section{Discussion}

David Graff: The EROS diagram showing how many stars we find as a function of period in both the LMC and the SMC does not show the standard metallicity effect. That is, it does not show that Cepheids of a particular period have a different luminosity from Cepheids with the same period, but different metallicity. It only shows that the instability strip extends to lower magnitudes for lower metallicities.

Bono: The EROS diagram shows that LMC Cepheids are systematically redder than SMC Cepheids. This effect implies that, in the $\log P-M_{v}$ plane, the LMC Cepheids are fainter than SMC Cepheids.

Norbert Langer: What is the improvement in the physics of your stellar models which allows you to obtain such an excellent agreement with observations, as compared to previous models.

Bono: The evolutionary tracks we adopted are canonical (i.e., no rotation, no mass-loss, no overshooting). For more details see Castellani, Chieffi, \& Straniero 1992). However, to account for the difference between canonical models and models constructed by adopting a mild convective core overshooting we computed a different set of nonlinear models by increasing the canonical luminosity by about 0.25 . With the exception of long period Cepheids the two sets of models present quite similar results concerning both the PL and PLC relations. Obviously, the zero-points are different. Concerning the pulsation models, the main improvement in the input physics is the inclusion of a nonlocal and timedependant treatment of convective transport (see Stellingwerf 1982; Bono \& Stellingwerf 1994).

Wolfgang Gieren: Just a comment: The best available data, both on LMC and on Galactive Cepheids, do not show any curvature of the Cepheid PL relation at the longest pulsation periods (see for example the Tauvir 1997 review paper at the Baltimore conference on the Extragalactic Distance Scale).

Bono: The EROS project in the paper by Sasselov et al. (1997) suggests that the slope of the PL relations changes toward long-period Cepheids $(\log P>1.8)$. Maybe these observations are in contradiction because the time spent inside the instability strip by high-mass stars is shorter than for lower masses. In our theoretical predictions we assume that the instability strip is uniformly filled.

Jean-Philip Beaulieu: I agree with you that one must include nonlinear, nonlocal time dependant turbulent convection in Cepheid envelopes. However, when you adopt one Mass-Luminosity relation for the metallicity of the Galaxy, LMC and SMC, you will not reproduce the position of the resonance centers (like the 10 day 2:1 resonance between the fundamental mode and second overtone) in the galaxies. You can use this constraint to have an idea about the difference in $M$, $\mathrm{L}$ between LMC, SMC, and the Galaxy.

Bono: This is a really interesting problem. We already obtained the Hertzsprung progression close to 10 days for LMC Cepheids. Owing to the small number of stellar masses we adopted we cannot say anything about the Hertzsprung progression in SMC and Galactic Cepheids. 